SISSA 40/2006/EP

\title{
Precision Measurement of Solar Neutrino Oscillation Parameters by a Long-Baseline Reactor Neutrino Experiment in Europe
}

\author{
S. T. Petcov ${ }^{a}$ and T. Schwetz \\ Scuola Internazionale Superiore di Studi Avanzati, and INFN, Sezione di Trieste, \\ Via Beirut 2-4, I-34014 Trieste, Italy
}

\begin{abstract}
We consider the determination of the solar neutrino oscillation parameters $\Delta m_{21}^{2}$ and $\theta_{12}$ by studying oscillations of reactor anti-neutrinos emitted by nuclear power plants (located mainly in France) with a detector installed in the Frejus underground laboratory. The performances of a water Čerenkov detector of $147 \mathrm{kt}$ fiducial mass doped with $0.1 \%$ of Gadolinium (MEMPHYS-Gd) and of a $50 \mathrm{kt}$ scale liquid scintillator detector (LENA) are compared. In both cases $3 \sigma$ uncertainties below $3 \%$ on $\Delta m_{21}^{2}$ and of about $20 \%$ on $\sin ^{2} \theta_{12}$ can be obtained after one year of data taking. The Gadolinium doped Super-Kamiokande detector (SK-Gd) in Japan can reach a similar precision if the SK/MEMPHYS fiducial mass ratio of 1 to 7 is compensated by a longer SK-Gd data taking time. Several years of reactor neutrino data collected by MEMPHYSGd or LENA would allow a determination of $\Delta m_{21}^{2}$ and $\sin ^{2} \theta_{12}$ with uncertainties of approximately $1 \%$ and $10 \%$ at $3 \sigma$, respectively. These accuracies are comparable to those that can be reached in the measurement of the atmospheric neutrino oscillation parameters $\Delta m_{31}^{2}$ and $\sin ^{2} \theta_{23}$ in long-baseline superbeam experiments.
\end{abstract}

\footnotetext{
${ }^{a}$ Also at: Institute of Nuclear Research and Nuclear Energy, Bulgarian Academy of Sciences, 1784 Sofia, Bulgaria.
} 


\section{Introduction}

The experiments with solar [1-3], atmospheric [4], reactor [5,6] and accelerator neutrinos $[8,9]$ have provided during the last several years compelling evidence for existence of neutrino oscillations caused by nonzero neutrino masses and neutrino mixing. The data imply the presence of $3-\nu$ mixing in the weak charged lepton current (see, e.g., [10]):

$$
\nu_{l \mathrm{~L}}=\sum_{j=1}^{3} U_{l j} \nu_{j \mathrm{~L}}, \quad l=e, \mu, \tau,
$$

where $\nu_{l \mathrm{~L}}$ are the flavour neutrino fields, $\nu_{j \mathrm{~L}}$ is the field of neutrino $\nu_{j}$ having a mass $m_{j}$ and $U$ is the Pontecorvo-Maki-Nakagawa-Sakata (PMNS) mixing matrix [11], $U \equiv U_{\text {PMNS. All }}$ currently existing $\nu$-oscillation data, except the data of the LSND experiment [12], can be described perfectly well assuming $3-\nu$ mixing in vacuum and we will consider this possibility in what follows. ${ }^{1}$

The PMNS matrix can be parametrized by three angles and, depending on whether the massive neutrinos $\nu_{j}$ are Dirac or Majorana particles, by one or three CP-violation (CPV) phases (see, e.g., [16]):

$$
U_{\mathrm{PMNS}}=V\left(\theta_{12}, \theta_{13}, \theta_{23}, \delta\right) \operatorname{diag}\left(1, e^{i \frac{\alpha}{2}}, e^{i \frac{\beta}{2}}\right)
$$

where $V\left(\theta_{12}, \theta_{13}, \theta_{23}, \delta\right)$ is a CKM-like matrix, $\delta$ is the Dirac CP-violating phase and $\alpha, \beta$ are two Majorana CPV phases $[17,18]$. If we standardly identify $\Delta m_{\odot}^{2}=\Delta m_{21}^{2} \equiv m_{2}^{2}-m_{1}^{2}>0$, where $\Delta m_{\odot}^{2}$ drives the solar neutrino oscillations, then $\Delta m_{\mathrm{A}}^{2}=\Delta m_{31}^{2} \cong \Delta m_{32}^{2}, \theta_{23}=\theta_{\mathrm{A}}$ and $\theta_{12}=\theta_{\odot}, \Delta m_{\mathrm{A}}^{2}, \theta_{\mathrm{A}}$ and $\theta_{\odot}$ being the $\nu$-mass squared difference and mixing angles responsible respectively for atmospheric and solar neutrino oscillations, while $\theta_{13}$ is the mixing angle constrained by the CHOOZ experiment [7]. The existing neutrino oscillation data allow us to determine $\Delta m_{21}^{2},\left|\Delta m_{31}^{2}\right|, \sin ^{2} \theta_{12}$ and $\sin ^{2} 2 \theta_{23}$ with a relatively good precision and to obtain rather stringent limits on $\sin ^{2} \theta_{13}$ (see, e.g., [19-21]). The best fit values and the $95 \%$ C.L. allowed ranges of $\Delta m_{21}^{2}, \sin ^{2} \theta_{12},\left|\Delta m_{31}^{2}\right|$ and $\sin ^{2} 2 \theta_{23}$ read:

$$
\begin{array}{ll}
\Delta m_{21}^{2}=8.0 \times 10^{-5} \mathrm{eV}^{2}, & \sin ^{2} \theta_{21}=0.31 \\
\left|\Delta m_{31}^{2}\right|=2.2 \times 10^{-3} \mathrm{eV}^{2}, & \sin ^{2} 2 \theta_{23}=1.0 \\
\Delta m_{21}^{2}=(7.3-8.5) \times 10^{-5} \mathrm{eV}^{2}, & \sin ^{2} \theta_{12}=(0.26-0.36), \\
\left|\Delta m_{31}^{2}\right|=(1.7-2.9) \times 10^{-3} \mathrm{eV}^{2}, & \sin ^{2} 2 \theta_{23} \geq 0.90 .
\end{array}
$$

A combined 3- $\nu$ oscillation analysis of the global data gives $[19,21]$

$$
\sin ^{2} \theta_{13}<0.027(0.044) \text { at } 95 \%(99.73 \%) \text { C.L. }
$$

Using the recently announced (but still unpublished) data from the MINOS experiment [9] in the analysis leads to a somewhat different best fit value and $95 \%$ allowed range of $\left|\Delta m_{31}^{2}\right|$

\footnotetext{
${ }^{1}$ In the LSND experiment indications for $\bar{\nu}_{\mu} \rightarrow \bar{\nu}_{e}$ oscillations with $\Delta m_{\mathrm{LSND}}^{2} \simeq 1 \mathrm{eV}^{2}$ were obtained. The minimal 4- $\nu$ mixing scheme which could incorporate the LSND indications for $\nu$-oscillations is strongly disfavored by the data [13]. A $\nu$-oscillation explanation of the LSND results might be possible assuming $5-\nu$ mixing [14]. The LSND results are being tested in the MiniBooNE experiment [15].
} 
and to a somewhat more stringent limit on $\sin ^{2} \theta_{13}[21]:\left|\Delta m_{31}^{2}\right|=2.6 \times 10^{-3} \mathrm{eV}^{2},\left|\Delta m_{31}^{2}\right|=$ $(2.2-3.0) \times 10^{-3} \mathrm{eV}^{2}$, and $\sin ^{2} \theta_{13}<0.025(0.040)$ at $95 \%(99.73 \%)$ C.L.

In spite of the enormous progress made in establishing the existence of neutrino oscillations driven by non-zero neutrino masses and mixing and in determining the pattern of neutrino mixing and the values of the two neutrino mass squared differences, our knowledge and understanding of neutrino mixing is rather limited at present (see, e.g., [10] for a detailed discussion of the current status of our ignorance about neutrino mixing). Future progress in the studies of neutrino mixing requires, in particular, the knowledge of the precise values of the parameters which drive the solar and the dominant atmospheric neutrino oscillations, $\Delta m_{21}^{2}, \sin ^{2} \theta_{12}, \Delta m_{31}^{2}$ and $\sin ^{2} \theta_{23}$ (see, e.g., [22]). The high precision measurement of these parameters is one of the main goals of the next generation of neutrino oscillation experiments. In the present article we discuss the possibility of a high precision measurement of the solar neutrino oscillation parameters $\Delta m_{21}^{2}$ and $\sin ^{2} \theta_{12}$ in an experiment studying the oscillations of reactor anti-neutrinos $\bar{\nu}_{e}$ with a "large scale" detector located in the Frejus underground laboratory in France.

The existing data allow a determination of $\Delta m_{21}^{2}$ and $\sin ^{2} \theta_{12}$ at $3 \sigma$ with an error of approximately $11 \%$ and $25 \%$, respectively. These parameters can and will be measured with higher precision in the future. The data from phase-III of the SNO experiment [3] using ${ }^{3} \mathrm{He}$ proportional counters for the neutral current rate measurement could lead to a reduction of the error in $\sin ^{2} \theta_{12}$ to $21 \%[23,24]$. If instead of $766.3 \mathrm{t}$ yr one uses simulated $3 \mathrm{kt}$ yr KamLAND data in the same global solar and reactor neutrino data analysis, the $3 \sigma$ errors in $\Delta m_{21}^{2}$ and $\sin ^{2} \theta_{12}$ diminish to $7 \%$ and $18 \%$ [24]. The most precise measurement of $\Delta m_{21}^{2}$, discussed so far in the literature, could be achieved [23] using Super-Kamiokande doped with $0.1 \%$ of Gadolinium (SK-Gd) for detection of reactor $\bar{\nu}_{e}$ [25]: the SK detector gets the same flux of reactor $\bar{\nu}_{e}$ as KamLAND and after 3 years of data-taking, $\Delta m_{21}^{2}$ could be determined with an error of $3.5 \%$ at $3 \sigma$ [23]. A dedicated reactor $\bar{\nu}_{e}$ experiment with a baseline $L \sim 60 \mathrm{~km}$, tuned to the minimum of the $\bar{\nu}_{e}$ survival probability, could provide the most precise determination of $\sin ^{2} \theta_{12}[26]$ : with statistics of $\sim 60 \mathrm{GW} \mathrm{kt}$ yr and a systematic error of $2 \%(5 \%), \sin ^{2} \theta_{12}$ could be measured with an accuracy of $6 \%(9 \%)$ at $3 \sigma$ [24]. The inclusion of the uncertainty in $\theta_{13}\left(\sin ^{2} \theta_{13}<0.05\right)$ in the analyzes increases the quoted errors by (1-3)\% to approximately $9 \%(12 \%)[24]$. The improved determination of $\Delta m_{21}^{2}$ and $\theta_{12}$ with KamLAND or dedicated post-KamLAND reactor neutrino experiments has been studied previously also in Refs. [27-31], whereas the potential improvements of the precision on these parameters from future solar neutrino experiments has been investigated, e.g., in Refs. [24, 26, 32,33].

MEMPHYS (MEgaton Mass PHYSics) [34] is a project for a mega ton scale water Čerenkov detector located in the Frejus underground laboratory at the border of France and Italy. It is similar to the UNO [35] project in the US and the future Hyper-Kamiokande [36] detector in Japan. Such detectors allow for a broad range of physics studies like nucleon decay, long-baseline accelerator neutrino oscillations, super nova neutrino detection, and oscillations of solar and atmospheric neutrinos. The MEMPHYS detector is considered as a far detector for neutrino beams produced at CERN located at a distance of $130 \mathrm{~km}$ from Frejus (see, e.g., Ref. [37]). A recent civil engineering pre-study indicates that MEMPHYS could be built with existing techniques as a modular detector consisting of three (up to five) modules (shafts), each having a fiducial mass of approximately $147 \mathrm{kt}$. 
In the present paper we consider the possibility that the water in one module of MEMPHYS is doped with $0.1 \%$ of Gadolinium (MEMPHYS-Gd), as it has been proposed originally for Super-K [25]. This allows a very efficient detection of electron anti-neutrinos through the reaction $\bar{\nu}_{e}+p \rightarrow e^{+}+n$ since the neutron can be tagged due to the high absorption cross section on Gadolinium. One module of MEMPHYS (147 kt) is about 6.5 times bigger than Super-K (22.5 kt), which increases correspondingly the potential for the various physics applications, such as detection of relic or galactic super nova neutrinos, see Ref. [25]. Here we explore the possibility of a precision measurement of the solar neutrino oscillation parameters $\Delta m_{21}^{2}$ and $\theta_{12}$ by studying the oscillations of electron anti-neutrinos emitted by the nuclear reactors located in the "neighborhood" of the Gd-doped MEMPHYS detector. We will compare, in particular, the precision on $\Delta m_{21}^{2}$ and $\theta_{12}$ which can be reached with the MEMPHYS-Gd detector with that obtainable with the Gd-doped Super-K detector (SK-Gd). The latter has been studied in detail in Ref. [23].

The water Čerenkov detectors typically do not have very good energy resolution, which is compensated to certain extent by their large mass. In what regards the energy resolution, the scintillator detectors such as KamLAND, perform significantly better. The LENA (Low Energy Neutrino Astronomy) detector [38] is a project for a large ( $\sim 50 \mathrm{kt})$ liquid scintillator detector, to be used for studies of relic and galactic super nova neutrinos, solar neutrinos, geoneutrinos, or proton decay. Since neutrinos from nuclear reactors constitute a background for the indicated measurements, some of the considered sites for LENA are rather far away from high concentrations of nuclear power plants. We consider in the following the possibility to place a LENA type detector in the Frejus laboratory (with many reactors relatively close by) and to use it for a high precision measurement of the oscillations of reactor anti-neutrinos.

\section{Reactor Neutrino Measurements with MEMPHYS-Gd and LENA Detectors at Frejus}

To calculate the flux of anti-neutrinos from reactors at a given position on the Earth, public available information on the nuclear power plants can be used [39]. A list of, and relevant data on reactors compiled from such sources has been kindly provided to us [40] for this study. To compare the reactor neutrino fluxes at Frejus with those at Kamioka, Japan, we consider first the effective reactor power at the detector, which is directly related to the total reactor $\bar{\nu}_{e}$ flux reaching the detector:

$$
W_{\mathrm{eff}}=\sum_{i} \frac{W_{i}^{\mathrm{th}}}{4 \pi L_{i}^{2}},
$$

where $W_{i}^{\text {th }}$ is the thermal power of the $i$ 'th reactor, $L_{i}$ is the distance to the detector, and the the sum runs over all contributing reactors. We find that for Kamioka $W_{\text {eff }}^{\text {Kamioka } \approx}$

$3.0 \mathrm{MW} \mathrm{km}^{-2} /(4 \pi)$, whereas at Frejus the reactor $\bar{\nu}_{e}$ flux is slightly higher with $W_{\text {eff }}^{\text {Frejus }} \approx$ $3.4 \mathrm{MW} \mathrm{km}^{-2} /(4 \pi)$.

The average distance traveled by reactor anti-neutrinos, $\langle L\rangle=\left(\sum_{i} W_{i}^{\text {th }} / L_{i}\right) /\left(4 \pi W_{\text {eff }}\right)$, is $188 \mathrm{~km}$ for Kamioka and $299 \mathrm{~km}$ for Frejus. Note that for $\Delta m_{21}^{2}=8 \times 10^{-5} \mathrm{eV}^{2}$ and a neutrino energy $E_{\nu} \sim 4 \mathrm{MeV}$ (corresponding to the maximum of the event rate in the absence of oscillations, see, e.g., the first article quoted in Ref. [28]) the first oscillation minimum 


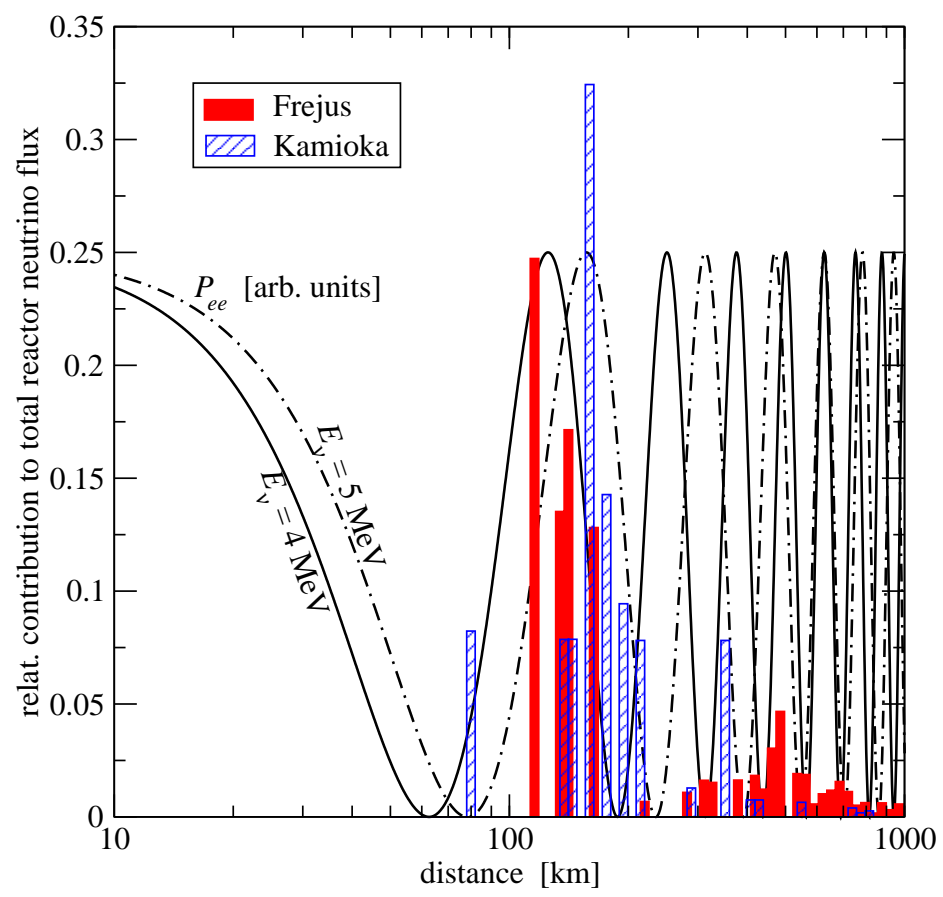

Figure 1: Relative contribution of different reactors to the total reactor neutrino flux at Frejus and Kamioka as a function of the distance to the reactor. Also shown are the $\bar{\nu}_{e}$ survival probabilities for $E_{\nu}=4 \mathrm{MeV}$ and $5 \mathrm{MeV}$ in arbitrary units.

of the reactor $\bar{\nu}_{e}$ survival probability is at approximately $60 \mathrm{~km}$. Therefore, the $\langle L\rangle$ for Frejus seems to be rather large for an optimal measurement of the oscillation parameters. However, the average distance can be misleading, and one should look at the $L$-distribution of the reactor $\bar{\nu}_{e}$ flux. In Fig. 1] we show the relative contribution of different reactors to the total reactor $\bar{\nu}_{e}$ flux at Frejus and Kamioka as a function of the baseline. It turns out that $67 \%$ of the total flux at Frejus originates from four reactors along the Rhone river located within a distance of $160 \mathrm{~km}$ from Frejus: Bugey at $115 \mathrm{~km}$ (25\%), Saint Alban at $133 \mathrm{~km}$ (13\%), Cruas at $142 \mathrm{~km}(16 \%)$, and Tricastin at $160 \mathrm{~km}(13 \%)^{2}$. Approximately $31 \%$ of the total flux comes from reactors distributed between $300 \mathrm{~km}$ and $1000 \mathrm{~km}$. In our analysis we include 56 reactors located at a distance $L<1000 \mathrm{~km}$, while the contributions of reactors at $L>1000 \mathrm{~km}$ from all around the world are summed to one "effective reactor" at $2500 \mathrm{~km}$ giving $2 \%$ of the total reactor $\bar{\nu}_{e}$ flux at Frejus.

The comparison of the $L$-distribution with the $\bar{\nu}_{e}$ survival probability in Fig. 1 shows that the 4 reactors providing the dominant part of the $\bar{\nu}_{e}$ flux at Frejus are located at distances which permit a rather precise study of reactor $\bar{\nu}_{e}$ oscillations. For a $\bar{\nu}_{e}$ energy $E_{\nu} \sim(4-5) \mathrm{MeV}$ they are located between the first and the second survival probability minima, and hence spectral information should provide a powerful tool to measure the oscillation parameters. In the case of Kamioka the $L$-distribution is rather centered around $\langle L\rangle \approx 190 \mathrm{~km}$. In Fig. 1 the important contribution to the $\bar{\nu}_{e}$ flux from the Kashiwazaki reactor complex located at approximately $160 \mathrm{~km}$ from Kamioka is clearly visible. For $E_{\nu} \sim 5 \mathrm{MeV}$ this distance corresponds to the first $\bar{\nu}_{e}$ survival probability maximum (see Ref. [27] for a detailed discussion).

\footnotetext{
${ }^{2}$ To use these four reactors for a measurement of $\theta_{12}$ and $\Delta m_{21}^{2}$ has been considered previously in Ref. [29].
} 
We calculate the observed prompt energy spectrum by

$$
\frac{d N}{d E_{p}}=\mathcal{N} \sum_{i} \frac{1}{4 \pi L_{i}^{2}} \int d E_{\nu} \sigma\left(E_{\nu}\right) \phi_{i}\left(E_{\nu}\right) P_{e e}\left(L_{i}, E_{\nu}\right) R\left(E_{p}^{\mathrm{tr}}, E_{p}\right),
$$

where the sum runs over the different reactors, $\sigma\left(E_{\nu}\right)$ is the cross section of the detection reaction $\bar{\nu}_{e}+p \rightarrow e^{+}+n, P_{e e}$ is the $\bar{\nu}_{e}$ survival probability, and $R\left(E_{p}^{\operatorname{tr}}, E_{p}\right)$ is the resolution function relating the "true prompt energy" $E_{p}^{\text {tr }}$ to the prompt energy $E_{p}$ observed in the detector, where $E_{p}^{\mathrm{tr}}$ is determined by the initial neutrino energy, $E_{p}^{\mathrm{tr}}=E_{\nu}-\left(m_{n}-m_{p}\right)+m_{e} \cong$ $E_{\nu}-0.8 \mathrm{MeV}$. We work with the total prompt energy visible in a scintillator detector (also if the actual detector considered is water Cerenkov) for the sake of comparison with KamLAND. For $R\left(E_{p}^{\mathrm{tr}}, E_{p}\right)$ we use a Gaussian resolution function with mean $E_{p}^{\mathrm{tr}}$, and a width of $44 \% / \sqrt{E_{p}[\mathrm{MeV}]}$ for MEMPHYS-Gd/SK-Gd and $10 \% / \sqrt{E_{p}[\mathrm{MeV}]}$ for LENA [38]. The energy resolution for MEMPHYS-Gd and SK-Gd is similar to the one reported by Super-K for the solar neutrino analysis (see Fig. 15 of the second paper in Ref. [2]).

In Eq. (6),$\phi_{i}\left(E_{\nu}\right)$ denotes the flux of $\bar{\nu}_{e}$ emitted by reactor $i$, which is given by

$$
\phi_{i}\left(E_{\nu}\right)=W_{i}^{\mathrm{th}} \sum_{\ell} \frac{f_{\ell}}{E_{\ell}} \phi_{\ell}\left(E_{\nu}\right)
$$

where $\ell={ }^{235} \mathrm{U},{ }^{238} \mathrm{U},{ }^{239} \mathrm{Pu},{ }^{241} \mathrm{Pu}$, labels the most important isotopes contributing to the $\bar{\nu}_{e}$ flux, $f_{\ell}$ is the relative contribution of the isotope $\ell$ to the total reactor power, and $E_{\ell}$ is the energy release per fission for the isotope $\ell$. In Eq. (7),$\phi_{\ell}\left(E_{\nu}\right)$ is the (energy differential) number of neutrinos emitted per fission by the isotope $\ell$, and we adopt the parameterization for the $\phi_{\ell}\left(E_{\nu}\right)$ from Ref. [41]. For the $f_{\ell}$ we take a typical isotope composition in a nuclear reactor of $[5]{ }^{235} \mathrm{U}:{ }^{238} \mathrm{U}:{ }^{239} \mathrm{Pu}:{ }^{241} \mathrm{Pu}=0.568: 0.297: 0.078: 0.057$, and we assume these ratios to be equal for all reactors.

In the calculations we use the 3-neutrino oscillation survival probability $P_{e e}$ which depends, in particular, on $\sin ^{2} \theta_{13}$ (see, e.g., [10]), and take into account the (small) Earth matter effect. In the case of absence of oscillations, $P_{e e}=1$, the number of events above a threshold $E_{\text {thr }}$ is given by $N_{\text {no osc }}=\mathcal{N} W_{\text {eff }} C$, where $W_{\text {eff }}$ has been defined in Eq. (15) and $C$ is an integral depending only on $E_{\text {thr }}$. For MEMPHYS-Gd we use a threshold for the prompt energy $E_{\mathrm{thr}}=3.0 \mathrm{MeV}$ (which corresponds to the value of $2.5 \mathrm{MeV}$ for the positron energy given in Ref. [25]), whereas for LENA we use $E_{\mathrm{thr}}=2.6 \mathrm{MeV}$ to eliminate the background from geo-neutrinos, as in the KamLAND oscillation analysis [5,6]. To determine the normalization constant $\mathcal{N}$ in Eq. (6) we use the prediction for $N_{\text {no osc }}$ in KamLAND [5], and then we scale it for each experiment taking into account that $\mathcal{N}$ is proportional to the measurement time and the number of free protons in the detector, as well as the different values of $W_{\text {eff }}$ and $E_{\mathrm{thr}}$. In Tab. 11 we summarize the most important characteristics of the considered detectors as simulated in our analysis, and we give the expected number of events in case of no oscillations.

To test the sensitivity of the experiments we divide the prompt energy spectrum in Eq. (6) into 20 bins between $3 \mathrm{MeV}$ and $12 \mathrm{MeV}$ for MEMPHYS-Gd and SK-Gd, and into 25 bins between $2.6 \mathrm{MeV}$ and $10 \mathrm{MeV}$ for LENA ${ }^{3}$. The data is simulated using as "true

\footnotetext{
${ }^{3}$ For low statistics data samples such as the present KamLAND one, a likelihood analysis [42] or equal bins in $1 / E_{p}$ [21] allows to extract an optimum of information. In the cases under study, however, the numbers of events are relatively high. Therefore, we simply take equal bins in $E_{p}$, with a bin size sufficiently smaller than the energy resolution to make sure that no information is lost due to the binning.
} 


\begin{tabular}{lrrrrrc}
\hline \hline experiment & \multicolumn{2}{c}{ fid. mass } & free protons & $E_{\text {thr }}$ & events/yr & energy resol. \\
\hline MEMPHYS-Gd & $147.0 \mathrm{kt}$ & $9.8 \times 10^{33}$ & $3.0 \mathrm{MeV}$ & 59980 & $44 \% / \sqrt{E_{p}[\mathrm{MeV}]}$ \\
LENA & $44.0 \mathrm{kt}$ & $2.3 \times 10^{33}$ & $2.6 \mathrm{MeV}$ & 16670 & $10 \% / \sqrt{E_{p}[\mathrm{MeV}]}$ \\
SK-Gd & $22.5 \mathrm{kt}$ & $1.5 \times 10^{33}$ & $3.0 \mathrm{MeV}$ & 8000 & $44 \% / \sqrt{E_{p}[\mathrm{MeV}]}$ \\
KamLAND & $0.41 \mathrm{kt}$ & $3.5 \times 10^{31}$ & $2.6 \mathrm{MeV}$ & 216 & $7.5 \% / \sqrt{E_{p}[\mathrm{MeV}]}$ \\
\hline \hline
\end{tabular}

Table 1: Summary of the input characteristics of the detectors MEMPHYS-Gd, LENA, and SK-Gd, used in our analysis. For comparison we show also the corresponding values for KamLAND [5]. The number of events/yr is calculated for no oscillations and using the reactor flux at Frejus for MEMPHYS-Gd and LENA, and at Kamioka for SK-Gd.

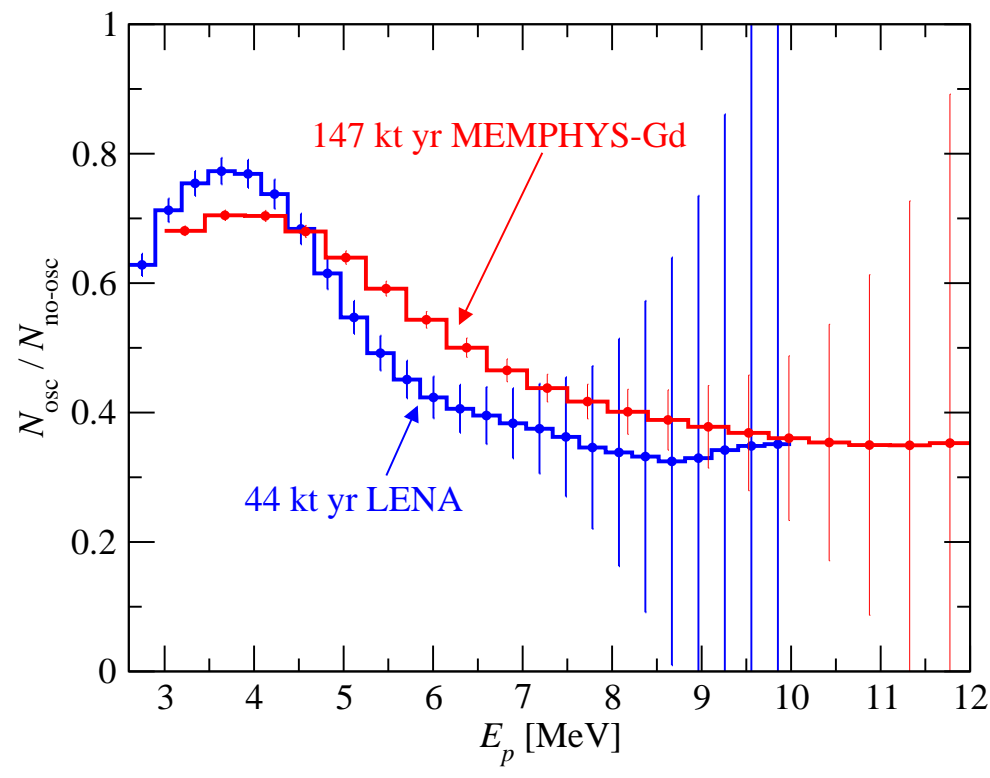

Figure 2: The ratio of the event spectra in positron energy in the case of oscillations with $\Delta m_{21}^{2}=$ $7.9 \times 10^{-5} \mathrm{eV}^{2}$ and $\sin ^{2} \theta_{12}=0.30$ and in the absence of oscillations, determined using one year data of MEMPHYS-Gd and LENA located at Frejus. The error bars correspond to $1 \sigma$ statistical error.

values" $\Delta m_{21}^{2}=7.9 \times 10^{-5} \mathrm{eV}^{2}$ and $\sin ^{2} \theta_{12}=0.30$. The latter correspond to the present best fit point obtained in a global neutrino oscillation analysis [21]. Then a $\chi^{2}$-analysis is performed to determine the allowed regions and the precision with which these parameters can be determined from the simulated data. In Fig. 2 we show the ratio of the number of events calculated by taking into account $\bar{\nu}_{e}$ oscillations with parameters indicated above to the number of events in the absence of oscillations, binned in prompt energy. The error bars correspond to $1 \sigma$ statistical errors for one year of (simulated) MEMPHYS-Gd and LENA data. The large number of events leads to a very precise measurement of the energy spectrum. The oscillatory signal in the spectrum is less pronounced in the MEMPHYS-Gd spectral "data" than in the analogous LENA "data" due to the worse energy resolution of the water Cerenkov detector. Nevertheless, as a consequence of the relatively high statistics, a clear signal of spectral distortion can still be observed with MEMPHYS-Gd. In the case of LENA spectral "data", an event maximum is clearly visible at $E_{p}=(3.5-4.0) \mathrm{MeV}$, which originates from the first oscillation maximum of the survival probability at $L \cong 160 \mathrm{~km}$ (see Fig. (1). In both cases the spectral information is crucial for the precise determination of the 


\begin{tabular}{lr}
\hline \hline systematic & value \\
\hline overall normalization (fully correlated) & $5 \%$ \\
thermal power of each reactor (uncorrelated) & $2 \%$ \\
energy scale uncertainty & $0.5 \%$ \\
prompt energy spectrum tilt & $2 \%$ \\
reactor neutrino flux & Ref. [41] \\
\hline \hline
\end{tabular}

Table 2: Systematical uncertainties and the default values adopted in our analysis.

oscillation parameters.

In the statistical analysis we take into account various systematical uncertainties as listed in Tab. 2. We include a $5 \%$ error on the overall normalization (e.g., from the uncertainty on the fiducial mass), a $2 \%$ uncertainty on the thermal power of each reactor (uncorrelated between the reactors), and the uncertainty on the anti-neutrino spectra $\phi_{\ell}\left(E_{\nu}\right)$ (normalization and shape) according to Ref. [41]. We take into account an uncertainty of $0.5 \%$ in the energy scale calibration of the detector. This value is motivated by the numbers given for the Super-K solar neutrino analysis (see second reference in [2]) and for the Double-Chooz reactor experiment [43]. Furthermore, we include a linear tilt in the prompt energy spectrum of $2 \%$, i.e., we allow the event number in the highest energy bin to shift by $2 \%$ with respect to the event number in the lowest energy bin with a linear interpolation for the intermediate bins. In the following section we will discuss in some detail how much our numerical results depend on the values adopted for the systematic uncertainties. In addition to these systematics we have tested also the effect of an uncertainty on the isotope compositions $f_{\ell}$ defined in Eq. (7). We have performed an analysis allowing the $f_{\ell}$ to vary independent for each reactor within $5 \%$, and found that the impact on the sensitivity to the neutrino oscillation parameters is negligible. Therefore, we keep the $f_{\ell}$ fixed in our standard analysis which significantly reduces the calculation time.

\section{Precision of the Determination of Neutrino Oscillation Para- meters}

In this Section we present results on the precision which can be reached in the measurement of $\Delta m_{21}^{2}$ and $\sin ^{2} \theta_{12}$ in the MEMPHYS-Gd and LENA experiments. Our findings are summarized in Fig. 3] and Tab. 3, where we compare the results which can be obtained using one year of data from MEMPHYS-Gd, LENA, and SK-Gd, with the present constraints from the global solar and KamLAND data $[19,21]$. In the table we give the relative uncertainty at $3 \sigma$ defined as

$$
\operatorname{spread}(x)=\frac{x^{\text {upper }}-x^{\text {lower }}}{x^{\text {upper }}+x^{\text {lower }}}
$$

where $x^{\text {upper }}\left(x^{\text {lower }}\right)$ is the upper (lower) bound of the quantity $x$ at $3 \sigma$.

We find that the water Cerenkov detector MEMPHYS-Gd and the scintillator detector LENA can provide very similar high precision determinations of $\Delta m_{21}^{2}$ and $\sin ^{2} \theta_{12}$. The lower mass of LENA is compensated by its better energy resolution. Already with one year of data, an uncertainty smaller than $3 \%$ at $3 \sigma$ can be obtained in the determination of 


\begin{tabular}{lcccc}
\hline \hline & \multicolumn{3}{c}{$\operatorname{spread}\left(\Delta m_{21}^{2}\right)$} & \multicolumn{2}{c}{$\operatorname{spread}\left(\sin ^{2} \theta_{12}\right)$} \\
time & $1 \mathrm{yr}$ & $7 \mathrm{yr}$ & $1 \mathrm{yr}$ & $7 \mathrm{yr}$ \\
\hline SK-Gd & $6.0 \%$ & $2.8 \%$ & $36.6 \%$ & $18.6 \%$ \\
MEMPHYS-Gd & $2.9 \%$ & $1.4 \%$ & $20.0 \%$ & $13.2 \%$ \\
LENA & $2.5 \%$ & $1.2 \%$ & $18.0 \%$ & $9.8 \%$ \\
\hline solar + KamLAND & \multicolumn{2}{c}{$11.3 \%$} & \multicolumn{2}{c}{$24.9 \%$} \\
\hline \hline
\end{tabular}

Table 3: The $3 \sigma$ uncertainty as defined in Eq. (8) in the determination of $\Delta m_{21}^{2}$ and $\sin ^{2} \theta_{12}$ in the experiments MEMPHYS-Gd and LENA at Frejus and SK-Gd at Kamioka after 1 and 7 years of data taking. For comparison we show also the current uncertainties in $\Delta m_{21}^{2}$ and $\sin ^{2} \theta_{12}$ [21].

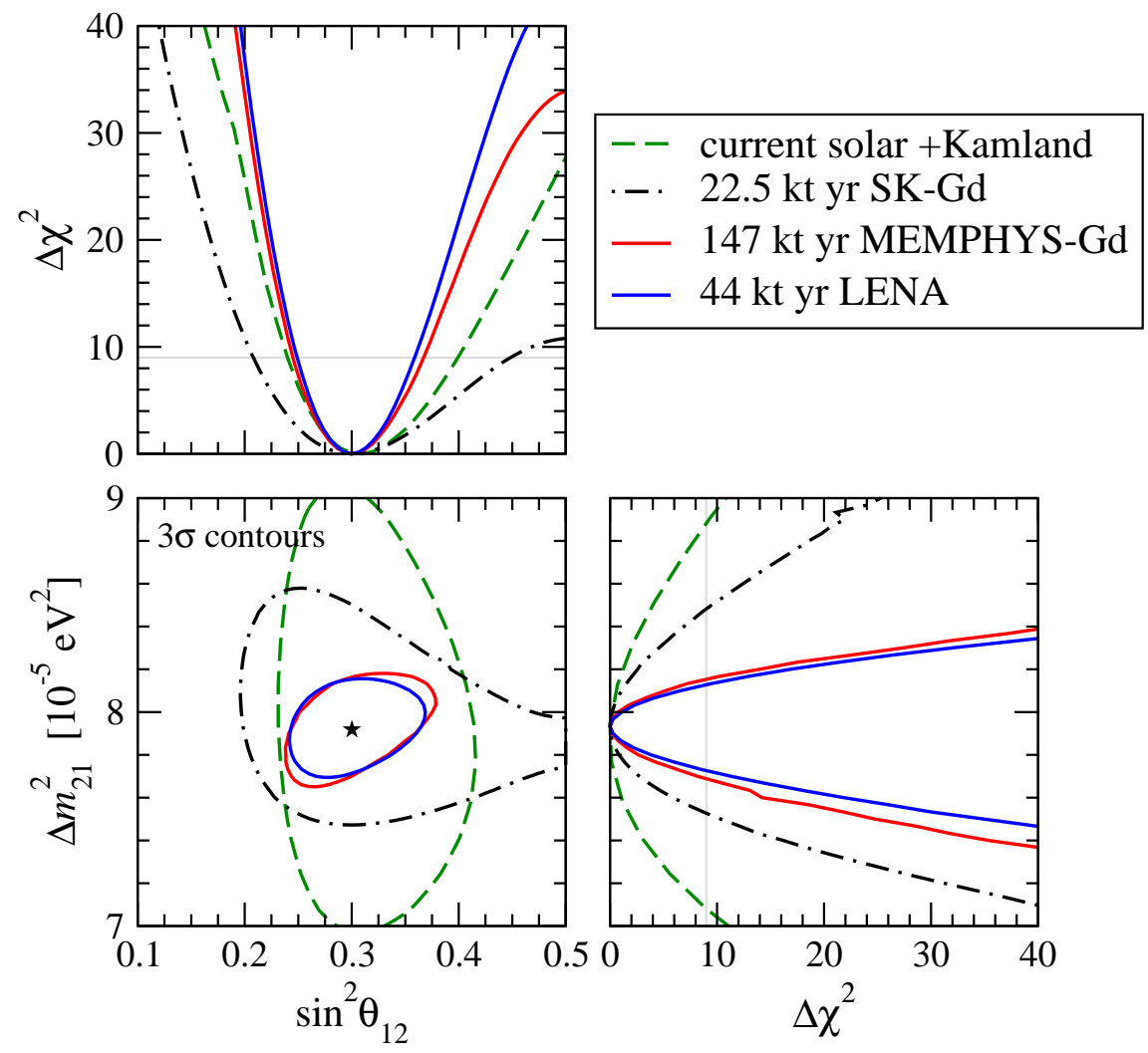

Figure 3: The accuracy of the determination of $\Delta m_{21}^{2}$ and $\sin ^{2} \theta_{12}$, which can be obtained using one year of data from MEMPHYS-Gd and LENA at Frejus, and from SK-Gd at Kamioka, compared to the current precision from solar neutrino and KamLAND data. We show the allowed regions at $3 \sigma$ (2 d.o.f.) in the $\Delta m_{21}^{2}-\sin ^{2} \theta_{12}$ plane, as well as the projections of the $\chi^{2}$ for each parameter.

$\Delta m_{21}^{2}$, while $\sin ^{2} \theta_{12}$ can be determined with an error of about $20 \%$ at $3 \sigma$. This precision is approximately by a factor two better than the precision that can be reached with one year of data from SK-Gd. The better precisions which can be obtained with the MEMPHYS-Gd detector compared to those that can be obtained with the SK-Gd detector are a consequence of the larger fiducial mass of MEMPHYS-Gd. As follows from Tab. [3 and Fig. [4 1 year of data from MEMPHYS-Gd and 7 years of data from SK-Gd (yielding approximately the same numbers of events in the two detectors, see Tab. (1) allow a determination of $\Delta m_{21}^{2}$ and $\sin ^{2} \theta_{12}$ with similar precisions. This shows, in particular, that both locations, Frejus and Kamioka, are very similar in what regards the power of the "surrounding" reactors and their 

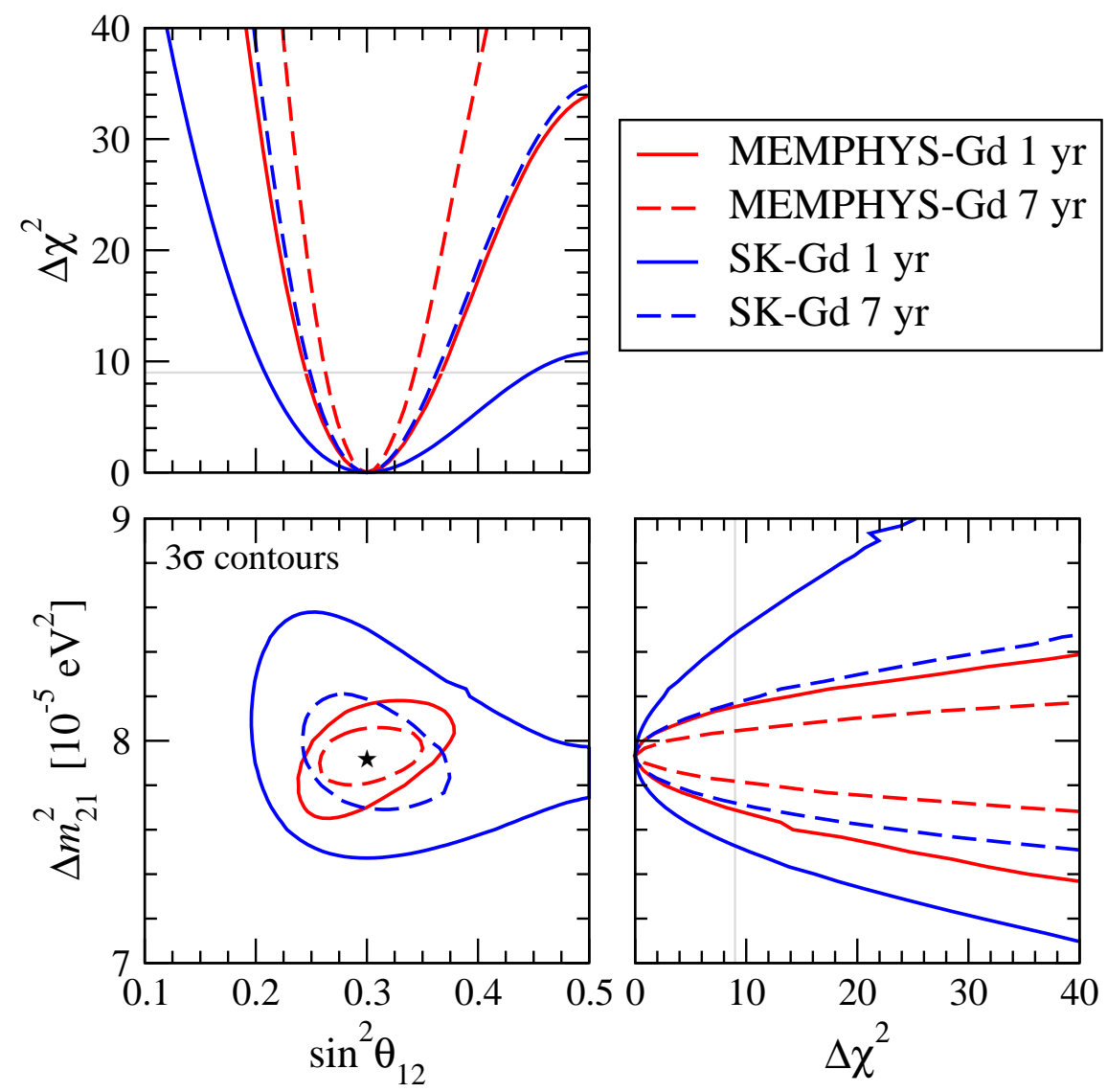

Figure 4: The allowed regions in the $\Delta m_{21}^{2}-\sin ^{2} \theta_{12}$ plane, obtained at $3 \sigma$ (2 d.o.f.) from $147 \mathrm{kt} \mathrm{yr}$ and $147 \times 7 \mathrm{kt}$ yr of MEMPHYS-Gd, and from $22.5 \mathrm{kt}$ yr and $22.5 \times 7=157.5 \mathrm{kt}$ yr of SK-Gd data. The projections of the $\chi^{2}$ for each parameter are also shown.

distance distribution. Thus, the two locations are equally suitable for the high precision measurements under discussion.

Ultimately, 7 years of data from MEMPHYS-Gd (LENA) would allow a determination of $\Delta m_{21}^{2}$ and $\sin ^{2} \theta_{12}$ with uncertainties of approximately $1.4 \%(1.2 \%)$ and $13 \%(10 \%)$ at $3 \sigma$, respectively. This precision is comparable to the precision which can be reached in the determination of the atmospheric neutrino oscillation parameters $\Delta m_{31}^{2}$ and $\sin ^{2} \theta_{23}$ by studying $\nu_{\mu}$ disappearance in the superbeam experiments T2HK in Japan or SPL from CERN to MEMPHYS (see e.g., Ref. [37] for a recent analysis). Hence, the reactor measurement could complete the program of the high precision determination of the parameters responsible for the leading solar and atmospheric neutrino oscillations.

We have also investigated whether the uncertainty in the knowledge of $\theta_{13}$ can have any effect on the precision of $\Delta m_{21}^{2}$ and $\theta_{12}$ determination in the experiments MEMPHYS-Gd and LENA under discussion. We show in Fig. 5 the results of a three-flavour neutrino oscillation analysis of 1 year simulated data in MEMPHYS-Gd, assuming that the true value of $\theta_{13}$ is zero. In the analysis $\sin ^{2} \theta_{13}$ was allowed to vary freely, however the information available at present has been included in the fit by adding the $\chi^{2}\left(\theta_{13}\right)$ obtained from current global data in Ref. [21]. In the panel for the $\sin ^{2} \theta_{12}-\Delta m_{21}^{2}$ projection we show also the allowed regions, obtained in a two-flavour analysis, i.e., for $\theta_{13}=0$, with black contours. Indeed, 


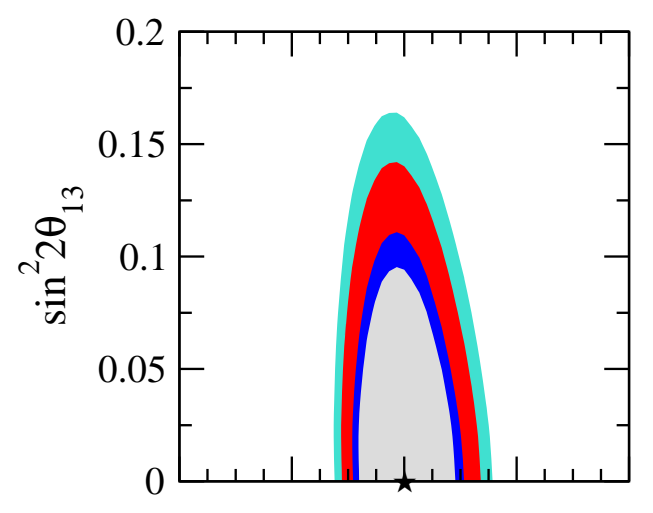

\section{7 kt yr MEMPHYS-Gd}
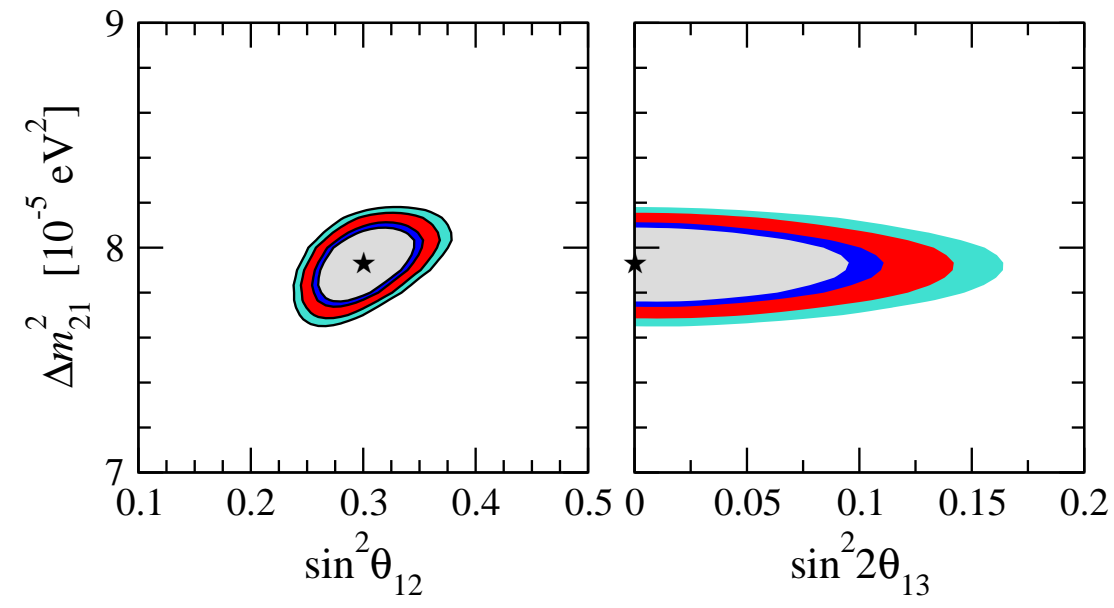

Figure 5: Allowed regions from a three-flavour neutrino oscillation analysis of $147 \mathrm{kt}$ yr data in MEMPHYS-Gd at 90\%, 95\%, 99\%, and 99.73\% CL (2 d.o.f.), projected onto the three different 2-dimensional parameter planes. The data are simulated for $\theta_{13}=0$. In the analysis $\theta_{13}$ was varied freely taking into account the constraint from the current global data as obtained in Ref. [21]. The allowed regions in the $\sin ^{2} \theta_{12}-\Delta m_{21}^{2}$ plane from a two-flavour analysis with $\theta_{13}$ fixed to zero (indicated with black contours) are identical to those obtained in the three-flavour analysis.

the regions within the black contours are practically identical to the shaded/colored regions corresponding to the three-flavour case. Therefore we can conclude that the uncertainty in the knowledge of $\theta_{13}$ does not affect the $\Delta m_{21}^{2}$ and $\theta_{12}$ measurements. As is visible in Fig. [5. there are no correlations of the leading parameters with $\theta_{13}$, since $\Delta m_{21}^{2}$ and $\theta_{12}$ are determined primarily from the data on the shape of the spectrum, which does not depend on $\theta_{13}$. We have checked that the above conclusions concerning the $\theta_{13}$-effects hold also for LENA and SK-Gd detectors.

Let us note that the sensitivity of MEMPHYS-Gd to $\theta_{13}$ on its own is rather poor. The constraint on $\sin ^{2} \theta_{13}$ appearing in Fig. 5 corresponds just to the bound from present global data, which is included in the analysis. Hence, the sensitivity of MEMPHYS-Gd is worse than the present bound. This is a consequence of the fact that a non-zero $\theta_{13}$ basically leads to a rather small (energy-independent) suppression of the total $\bar{\nu}_{e}$ flux, which is unobservable due to the relatively large uncertainties in the overall normalization.

Since we are discussing here very high statistics measurements, a careful investigation of the impact of systematical uncertainties is necessary. In Fig. [6 we show how our results for the accuracies of the oscillation parameters depend on the assumptions adopted for the 
$\square$ MEMPHYS-Gd (1yr) $\square$ LENA (1yr)

WII SK-Gd (7yr)

\begin{tabular}{|l|}
\hline All systematics \\
\hline Reactor power \\
\hline Energy scale \\
\hline Spectrum tilt \\
\hline Normalization \\
\hline
\end{tabular}
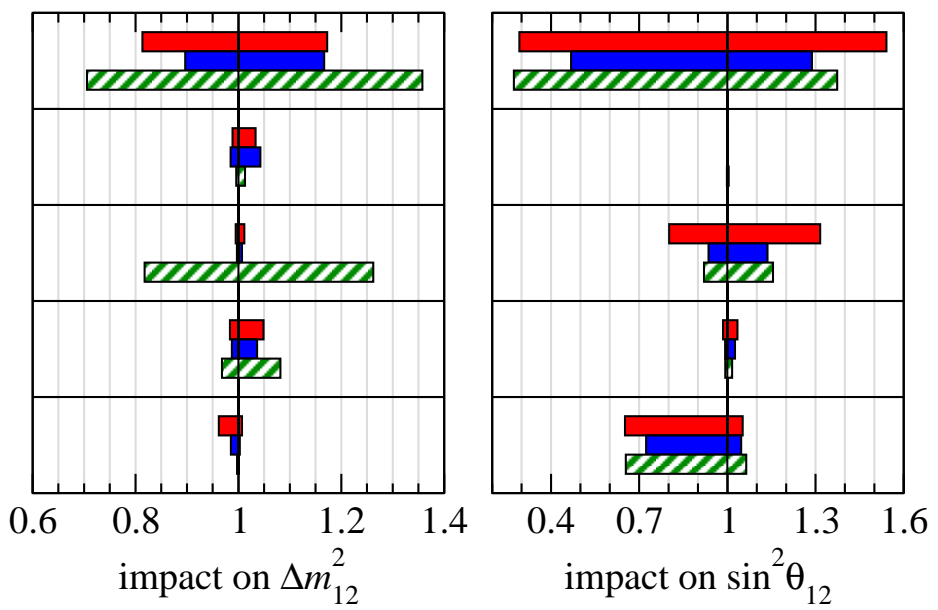

Figure 6: Impact of systematical uncertainties. We show the relative change in the $3 \sigma$ intervals of $\Delta m_{21}^{2}$ and $\sin ^{2} \theta_{12}$ obtained by switching off (left edges of the bars) and doubling (right edges) all systematics simultaneously, the uncertainties on the thermal power of the 4 most important reactors ("Reactor power"), the energy scale uncertainty, the prompt energy spectrum tilt, and the overall normalization error.

systematical errors. In particular, we consider the impact of uncertainties on the thermal power of the 4 most important reactors, the energy scale uncertainty, the prompt energy spectrum tilt, the overall normalization error, as well as all systematical errors in total. To check the impact of these uncertainties we show in Fig. 6] the ratios $\delta_{0} / \delta_{\text {std }}$ and $\delta_{2} / \delta_{\text {std }}$, where $\delta_{0(2)}$ is the $3 \sigma$ range for $\Delta m_{21}^{2}$ or $\sin ^{2} \theta_{12}$ if the systematical error of interest is set to zero (is doubled with respect to its standard value), and $\delta_{\text {std }}$ is the $3 \sigma$ range using our standard values according to Tab. 2.

The left edges of the bars in the row denoted by "All systematics" in Fig. 6 correspond to statistical errors only. In this ideal case the $\Delta m_{21}^{2}$ accuracy is improved by about $20 \%$ for MEMPHYS-Gd, 10\% for LENA, and 30\% for SK-Gd with respect to our standard choice for the systematics, whereas the precision on $\sin ^{2} \theta_{12}$ is improved for all experiments by more than a factor of 2 . For the $\Delta m_{21}^{2}$ measurement the individual systematics have only a minor impact (with the exception of a $\sim 20 \%$ effect of the energy scale uncertainty in SK-Gd). For the measurement of $\sin ^{2} \theta_{12}$ the overall normalization and the energy scale (especially for MEMPHYS-Gd) are important. Note that the uncertainty on the thermal reactor power has a negligible impact on the accuracies. Therefore, it seems not to be possible to improve the precision on $\Delta m_{21}^{2}$ and $\theta_{12}$ by installing near detectors close to the reactors dominating the $\bar{\nu}_{e}$ flux.

In summary, systematic uncertainties are an important factor in the experiments under consideration. Especially the determination of the mixing angle depends on the values of systematic errors. The overall effect emerges from an interplay of the various sources of uncertainties included in our analysis, and therefore, to obtain an improvement in the precision of the oscillation parameters several of the systematic errors listed in Tab. 2] should be decreased. 


\section{Conclusions}

We have investigated the possibility of a high precision determination of the solar neutrino oscillation parameters $\Delta m_{21}^{2}$ and $\theta_{12}$ in a long-baseline reactor neutrino experiment, located in the Frejus underground laboratory. Approximately $67 \%$ of the total reactor $\bar{\nu}_{e}$ flux at Frejus originates from four nuclear power plants in the Rhone valley, located at distances between $115 \mathrm{~km}$ and $160 \mathrm{~km}$ from Frejus. The indicated baselines are particularly suitable for the study of the $\bar{\nu}_{e}$ oscillations driven by $\Delta m_{21}^{2}$ - they are similar to those exploited in the KamLAND experiment in Japan. Approximately $31 \%$ of the total flux $\bar{\nu}_{e}$ at Frejus comes from reactors distributed between $300 \mathrm{~km}$ and $1000 \mathrm{~km}$ from Frejus. In our analysis we include 56 reactors located at a distance $L<1000 \mathrm{~km}$, while the contributions of reactors at $L>1000 \mathrm{~km}$ from all around the world are summed to one "effective reactor" at $2500 \mathrm{~km}$ giving $2 \%$ of the total reactor $\bar{\nu}_{e}$ flux at Frejus. The Frejus underground laboratory is under consideration as a possible site for a mega ton scale water Cerenkov detector MEMPHYS which, among other physics applications, may serve as a far detector for a neutrino beam produced at CERN. In the present article we have assumed that the water of one module of MEMPHYS having a fiducial mass of $147 \mathrm{kt}$, is doped with $0.1 \%$ Gadolinium (MEMPHYSGd), which will allow, in principle, a high precision study of reactor $\bar{\nu}_{e}$ oscillations. As an alternative detector technology, we have considered a $50 \mathrm{kt}$ scale liquid scintillator detector, as discussed in the LENA proposal, which can be viewed as a considerably larger version of the present KamLAND or Borexino detectors.

The analysis performed by us shows that each of the two detectors-MEMPHYS-Gd and LENA, if placed at Frejus, would allow a very precise determination of the solar neutrino oscillation parameters $\Delta m_{21}^{2}$ and $\sin ^{2} \theta_{12}$ : with one year of reactor $\bar{\nu}_{e}$ data taken at Frejus (by any of the two detectors), the $3 \sigma$ uncertainties on $\Delta m_{21}^{2}$ and $\sin ^{2} \theta_{12}$ can be reduced respectively to less than $3 \%$ and to approximately 20\%. The Gadolinium doped SuperKamiokande detector (SK-Gd) in Japan can reach a similar precision if the SK/MEMPHYS fiducial mass ratio of 1 to 7 is compensated by a longer SK-Gd data taking time. Several years of reactor $\bar{\nu}_{e}$ data collected by MEMPHYS-Gd or LENA would allow a determination of $\Delta m_{21}^{2}$ and $\sin ^{2} \theta_{12}$ with uncertainties of approximately $1 \%$ and $10 \%$ at $3 \sigma$, respectively. We have shown also that the uncertainty associated with the CHOOZ mixing angle $\theta_{13}$ has practically no impact on the measurements of the solar neutrino oscillation parameters in the experiments discussed by us, and we have investigated in some detail the effects of various systematical uncertainties on the precision of the determination of $\Delta m_{21}^{2}$ and $\sin ^{2} \theta_{12}$ in these experiments.

The accuracies on the solar oscillation parameters, which can be obtained in the high statistics experiments considered here are comparable to those that can be reached for the atmospheric neutrino oscillation parameters $\Delta m_{31}^{2}$ and $\sin ^{2} \theta_{23}$ in future long-baseline superbeam experiments like T2HK in Japan or SPL from CERN to MEMPHYS. Hence, such reactor measurements would complete the program of the high precision determination of the leading neutrino oscillation parameters.

Acknowledgments. We are grateful to Alessandra Tonazzo and Michael Wurm for providing us information on nuclear power plants, and we thank Jean-Eric Campagne and Michael Wurm for useful communications on the MEMPHYS and LENA detectors. This 
work was supported in part by the Italian MIUR and INFN under the programs "Fisica Astroparticellare" (S.T.P.). The work of T.S. is supported by a "Marie Curie Intra-European Fellowship within the 6th European Community Framework Program."

\section{References}

[1] B. T. Cleveland et al., Astrophys. J. 496 (1998) 505; J.N. Abdurashitov et al. [SAGE Collaboration], J. Exp. Theor. Phys. 95 (2002) 181 astro-ph/0204245; T. Kirsten et al. [GALLEX and GNO Collaborations], Nucl. Phys. B (Proc. Suppl.) 118 (2003) 33; C. Cattadori, Talk given at Neutrino04, June 14-19, 2004, Paris, France.

[2] Super-K Collaboration, S. Fukuda et al., Phys. Lett. B 539 (2002) 179 hep-ex/0205075; J. Hosaka et al. hep-ex/0508053.

[3] SNO Collaboration, Q.R. Ahmad et al., Phys. Rev. Lett. 89, 011302 (2002) nucl-ex/0204009; B. Aharmim et al., Phys. Rev. C 72, 055502 (2005) nucl-ex/0502021.

[4] Super-K Collaboration, Y. Fukuda et al., Phys. Rev. Lett. 81 (1998) 1562 hep-ex/9807003; Y. Ashie et al., Phys. Rev. D 71 (2005) 112005 hep-ex/0501064.

[5] K. Eguchi et al. [KamLAND Collaboration], Phys. Rev. Lett. 90 (2003) 021802 hep-ex/0212021.

[6] T. Araki et al. [KamLAND Collaboration], Phys. Rev. Lett. 94, 081801 (2005) hep-ex/0406035.

[7] M. Apollonio et al. [CHOOZ Collaboration], Phys. Lett. B 466, 415 (1999) hep-ex/9907037; Eur. Phys. J. C 27, 331 (2003) hep-ex/0301017.

[8] K2K Collaboration, E. Aliu et al., Phys. Rev. Lett. 94, 081802 (2005) hep-ex/0411038; M. H. Ahn et al., hep-ex/0606032.

[9] E.Ables et al. [MINOS Collaboration] FERMILAB-PROPOSAL-P-875; D.A.Petyt, talk at Fermilab, 30 march 2006, http://www-numi.fnal.gov/talks/results06.html; J. Nelson, Talk at Neutrino 2006, 13-19 June 2006, Santa Fe, New Mexico, http://neutrinosantafe06.com/

[10] S. T. Petcov, Nucl. Phys. Proc. Suppl. 143, 159 (2005) hep-ph/0412410; Phys. Scripta T121, 94 (2005) hep-ph/0504166.

[11] B. Pontecorvo, J. Exptl. Theoret. Phys. 33 (1957) 549. [Sov. Phys. JETP 6 (1958) 429]; J. Exptl. Theoret. Phys. 34 (1958) 247 [Sov. Phys. JETP 7 (1958) 172]; Z. Maki, M. Nakagawa and S. Sakata, Prog. Theor. Phys. 28 (1962) 870.

[12] A. Aguilar et al. [LSND Collaboration], Phys. Rev. D 64, 112007 (2001) hep-ex/0104049.

[13] M. Maltoni et al., Nucl. Phys. B 643, 321 (2002) hep-ph/0207157. 
[14] M. Sorel, J. Conrad and M. Shaevitz, Phys. Rev. D70 (2004) 073004 hep-ph/0305255.

[15] MiniBooNE Collaboration, A. A. Aguilar-Arevalo, hep-ex/0408074 R. Tayloe et al., Nucl. Phys. Proc. Suppl. 118, 157 (2003); Heather L. Ray et al., hep-ex/0411022

[16] S. M. Bilenky, S. Pascoli and S. T. Petcov, Phys. Rev. D 64, 053010 (2001) hep-ph/0102265. S. Pascoli and S.T. Petcov, hep-ph/0308034.

[17] S.M. Bilenky, J. Hosek and S.T. Petcov, Phys. Lett. B 94 (1980) 495.

[18] M. Doi et al., Phys. Lett. B 102 (1981) 323; J. Schechter and J. W. F. Valle, Phys. Rev. D 22 (1980) 2227.

[19] A. Bandyopadhyay et al., Phys. Lett. B 608, 115 (2005) hep-ph/0406328]; A. Bandyopadhyay et al., 2005 (unpublished); see also A. Bandyopadhyay et al., Phys. Lett. B 583, 134 (2004) hep-ph/0309174.

[20] T. Schwetz, Acta Phys. Polon. B 36 (2005) 3203 hep-ph/0510331.

[21] T. Schwetz, hep-ph/0606060 M. Maltoni et al., New J. Phys. 6 (2004) 122, see hep-ph/0405172 v5 for updated results.

[22] M. Freund, P. Huber and M. Lindner, Nucl. Phys. B 615 (2001) 331 hep-ph/0105071; A. Donini, D. Meloni and S. Rigolin, Eur. Phys. J. C 45, 73 (2006) hep-ph/0506100|.

[23] S. Choubey and S.T. Petcov, Phys. Lett. B 594 (2004) 333 hep-ph/0404103.

[24] A. Bandyopadhyay et al., Phys. Rev. D 72 (2005) 072002 hep-ph/0410283.

[25] J. Beacom and M. Vagins, Phys. Rev. Lett. 93, 171101 (2004) hep-ph/0309300.

[26] A. Bandyopadhyay et al., Phys. Rev. D 67 (2003) 113011 hep-ph/0302243.

[27] A. Bandyopadhyay et al., Phys. Lett. B 581 (2004) 62 hep-ph/0309236.

[28] S. T. Petcov and M. Piai, Phys. Lett. B 533 (2002) 94 hep-ph/0112074; S. Schonert, T. Lasserre and L. Oberauer, Astropart. Phys. 18 (2003) 565 hep-ex/0203013; S. Choubey, S. T. Petcov and M. Piai, Phys. Rev. D 68 (2003) 113006 hep-ph/0306017.

[29] C. Bouchiat, hep-ph/0304253.

[30] H. Minakata et al., Phys. Rev. D 71 (2005) 013005 hep-ph/0407326.

[31] J. F. Kopp et al., hep-ph/0606151.

[32] J.N. Bahcall and C. Peña-Garay, JHEP 0311 (2003) 004 hep-ph/0305159.

[33] A. Kopylov and V. Petukhov, hep-ph/0308004.

[34] A. de Bellefon et al., MEMPHYS: A large scale water Čerenkov detector at Fréjus, Contribution to the CERN strategic committee, http://apc-p7.org/APC_CS/Experiences/MEMPHYS/ 
[35] C. K. Jung, Feasibility of a next generation underground water Cherenkov detector: UNO, hep-ex/0005046.

[36] K. Nakamura, Int. J. Mod. Phys. A 18 (2003) 4053.

[37] J. E. Campagne, M. Maltoni, M. Mezzetto and T. Schwetz, hep-ph/0603172.

[38] L. Oberauer, F. von Feilitzsch and W. Potzel, Nucl. Phys. Proc. Suppl. 138 (2005) 108; T. Marrodan Undagoitia et al., Prog. Part. Nucl. Phys. 57 (2006) 283 hep-ph/0605229; LENA webpage: http://www.e15.physik.tu-muenchen.de/research/lena.html

[39] Information on world wide nuclear power plants is available e.g., at the webpages of the International Nuclear Safety Center (http://www.insc.anl.gov/) or the Nuclear Training Center (http://www.icjt.org).

[40] A. Tonazzo and M. Wurm, private communication.

[41] P. Huber and T. Schwetz, Phys. Rev. D 70 (2004) 053011 hep-ph/0407026.

[42] T. Schwetz, Phys. Lett. B 577 (2003) 120 hep-ph/0308003.

[43] F. Ardellier et al. [Double-Chooz Collaboration], hep-ex/0606025. 